\title{
Quantum Programs as Kleisli Maps
}

\author{
Abraham Westerbaan \\ Radboud University Nijmegen \\ bram@westerbaan. name
}

Furber and Jacobs have shown in their study of quantum computation that the category of commutative $C^{*}$-algebras and $P U$-maps (positive linear maps which preserve the unit) is isomorphic to the Kleisli category of a comonad on the category of commutative $C^{*}$-algebras with $M I U$-maps (linear maps which preserve multiplication, involution and unit). [3]

In this paper, we prove a non-commutative variant of this result: the category of $C^{*}$-algebras and PU-maps is isomorphic to the Kleisli category of a comonad on the subcategory of MIU-maps.

A variation on this result has been used to construct a model of Selinger and Valiron's quantum lambda calculus using von Neumann algebras. [1]

The semantics of a non-deterministic program that takes two bits and returns three bits can be described as a multimap (= binary relation) from $\{0,1\}^{2}$ to $\{0,1\}^{3}$. Similarly, a program that takes two qubits and returns three qubits can be modelled as a positive linear unit-preserving map from $\mathrm{M}_{2} \otimes \mathrm{M}_{2} \otimes \mathrm{M}_{2}$ to $\mathrm{M}_{2} \otimes \mathrm{M}_{2}$, where $\mathrm{M}_{2}$ is the $C^{*}$-algebra of $2 \times 2$-matrices over $\mathbb{C}$.

More generally, the category Set $_{\text {multi }}$ of multimaps between sets models non-deterministic programs (running on an ordinary computer), while the opposite of the category $\mathbf{C}_{\mathrm{PU}}^{*}$ of $P U$-maps (positive linear unit-preserving maps) between $C^{*}$-algebras models programs running on a quantum computer. (When we write " $C^{*}$-algebra" we always mean " $C^{*}$-algebra with unit".)

A multimap from $\{0,1\}^{2}$ to $\{0,1\}^{3}$ is simply a map from $\{0,1\}^{2}$ to $\mathscr{P}\left(\{0,1\}^{3}\right)$. In the same line $\mathbf{S e t}_{\text {multi }}$ is (isomorphic to) the Kleisli category of the powerset monad $\mathscr{P}$ on Set. What about $\mathbf{C}_{\mathrm{PU}}^{*}$ ?

We will show that there is a monad $\Omega$ on $\left(\mathbf{C}_{\mathrm{MIU}}^{*}\right)^{\text {op }}$, the opposite of the category $\mathbf{C}_{\mathrm{MIU}}^{*}$ of $C^{*}$-algebras and MIU-maps (linear maps that preserve the multiplication, involution and unit), such that $\left(\mathbf{C}_{\mathrm{PU}}^{*}\right)^{\text {op }}$ is isomorphic to the Kleisli category of $\Omega$. We say that $\left(\mathbf{C}_{\mathrm{PU}}^{*}\right)^{\text {op }}$ is Kleislian over $\left(\mathbf{C}_{\mathrm{MIU}}^{*}\right)^{\mathrm{op}}$. So in the same way we add non-determinism to Set by the powerset monad $\mathscr{P}$ yielding $\mathbf{S e t}_{\text {multi }}$, we can obtain $\left(\mathbf{C}_{\mathrm{PU}}^{*}\right)^{\text {op }}$ from $\left(\mathbf{C}_{\mathrm{MIU}}^{*}\right)^{\text {op }}$ by a monad $\Omega$.

Let us spend some words on how we obtain this monad $\Omega$. Note that since every positive element of a $C^{*}$-algebra $\mathscr{A}$ is of the form $a^{*} a$ for some $a \in \mathscr{A}$ any MIU-map will be positive. Thus $\mathbf{C}_{\text {MIU }}^{*}$ is a subcategory of $\mathbf{C}_{\mathrm{PU}}^{*}$. Let $U: \mathbf{C}_{\mathrm{MIU}}^{*} \longrightarrow \mathbf{C}_{\mathrm{PU}}^{*}$ be the embedding.

In Section 1 we will prove that $U$ has a left adjoint $F: \mathbf{C}_{\mathrm{PU}}^{*} \longrightarrow \mathbf{C}_{\mathrm{MIU}}^{*}$, see Theorem 5 . This adjunction gives us a comonad $\Omega:=F U$ on $\mathbf{C}_{\mathrm{MIU}}^{*}$ (which is a monad on $\left(\mathbf{C}_{\mathrm{MIU}}^{*}\right)^{\text {op }}$ ) with the same counit as the adjunction. The comultiplication $\delta$ is given by $\delta_{\mathscr{A}}=F \eta_{U \mathscr{A}}$ for every object $\mathscr{A}$ from $\mathbf{C}_{\mathrm{MIU}}^{*}$ where $\eta$ is the unit of the adjunction between $F$ and $U$.

In Section 2 we will prove that $\left(\mathbf{C}_{\mathrm{PU}}^{*}\right)^{\text {op }}$ is isomorphic to $\mathscr{K} \ell(F U)$ if $F U$ is considered a monad on $\left(\mathbf{C}_{\mathrm{MIU}}^{*}\right)^{\text {op }}$. In fact, we will prove that the comparison functor $L: \mathscr{K} \ell(F U) \longrightarrow\left(\mathbf{C}_{\mathrm{PU}}^{*}\right)^{\text {op }}$ (which sends a MIU-map $f: F U \mathscr{A} \longrightarrow \mathscr{B}$ to $\left.U f \circ \eta_{U \mathscr{A}}: U \mathscr{A} \longrightarrow U \mathscr{B}\right)$ is an isomorphism, see Corollary 10 .

The method used to show that $\left(\mathbf{C}_{\mathrm{PU}}^{*}\right)^{\text {op }}$ is Kleislian over $\left(\mathbf{C}_{\mathrm{MIU}}^{*}\right)^{\text {op }}$ is quite general and it will be obvious that many variations on $\left(\mathbf{C}_{\mathrm{PU}}^{*}\right)^{\text {op }}$ will be Kleislian over $\left(\mathbf{C}_{\mathrm{MIU}}^{*}\right)^{\text {op }}$ as well, such as the opposite of the category of subunital completely positive linear maps between $C^{*}$-algebras. The flip-side of this generality is that we discover preciously little about the monad $\Omega$ which leaves room for future inquiry (see Section 3).

R. Duncan and C. Heunen (Eds.): Quantum Physics and Logic (QPL) 2016 EPTCS 236, 2017, pp. 215-228 doi 10.4204/EPTCS.236.14
() A.A. Westerbaan

This work is licensed under the Creative Commons Attribution License. 
We will also see that the opposite $\left(\mathbf{W}_{\mathrm{NCPs}}^{*}\right)^{\text {op }}$ of the category of normal completely positive subunital maps between von Neumann algebras is Kleislian over the subcategory $\left(\mathbf{W}_{\mathrm{NMIU}}^{*}\right)^{\text {op }}$ of normal unital *homomorphisms. This fact is used in [1] to construct an adequate model of Selinger and Valiron's quantum lambda calculus using von Neumann algebras.

\section{The Left Adjoint}

In Theorem 5 we will show that $U$ has a left adjoint, $F: \mathbf{C}_{\mathrm{MIU}}^{*} \rightarrow \mathbf{C}_{\mathrm{PU}}^{*}$, using a quite general method. As a result we do not get any "concrete" information about $F$ in the sense that while we will learn that for every $C^{*}$-algebra $\mathscr{A}$ there exists an arrow $\rho: \mathscr{A} \rightarrow U F \mathscr{A}$ which is initial from $\mathscr{A}$ to $U$ we will learn nothing more about $\rho$ than this. Nevertheless, for some (very) basic $C^{*}$-algebras $\mathscr{A}$ we can describe $F \mathscr{A}$ directly, as is shown below in Example 1 , 3 .

Example 1. Let us start easy: $\mathbb{C}$ will be mapped to itself by $F$, that is: the identity $\rho: \mathbb{C} \longrightarrow U \mathbb{C}$ is an initial arrow from $\mathbb{C}$ to $U(-)$.

Indeed, let $\mathscr{A}$ be a $C^{*}$-algebra and let $\sigma: \mathbb{C} \rightarrow U \mathscr{A}$ be a PU-map. Then $\sigma$ must be given by $\sigma(\lambda)=\lambda \cdot 1$ for $\lambda \in \mathbb{C}$, where 1 is the identity of $\mathscr{A}$. Thus $\sigma$ is a MIU-map as well. Hence there is a unique MIU-map $\hat{\sigma}: \mathbb{C} \rightarrow \mathscr{A}$ (namely $\hat{\sigma}=\sigma$ ) such that $\hat{\sigma} \circ \rho=\sigma$. ( $\mathbb{C}$ is initial in both $\mathbf{C}_{\mathrm{MIU}}^{*}$ and $\mathbf{C}_{\mathrm{PU}}^{*}$ )

Example 2. The image of $\mathbb{C}^{2}$ under $F$ will be the $C^{*}$-algebra $C[0,1]$ of continuous functions from $[0,1]$ to $\mathbb{C}$. As will become clear below, this is very much related to the familiar functional calculus for $C^{*}$ algebras: given an element $a$ of a $C^{*}$-algebra $\mathscr{A}$ with $0 \leq a \leq 1$ and $f \in C[0,1]$ we can make sense of " $f(a)$ ", as an element of $\mathscr{A}$.

The map $\rho: \mathbb{C}^{2} \longrightarrow U C[0,1]$ given by, for $\lambda, \mu \in \mathbb{C}, x \in[0,1]$,

$$
\rho(\lambda, \mu)(x)=\lambda x+\mu(1-x)
$$

is an initial arrow from $\mathbb{C}^{2}$ to $U$.

Let $\sigma: \mathbb{C}^{2} \rightarrow U \mathscr{A}$ be a PU-map. We must show that there is a unique MIU-map $\bar{\sigma}: C[0,1] \rightarrow \mathscr{A}$ such that $\sigma=\bar{\sigma} \circ \rho$.

Writing $a:=\sigma(1,0)$, we have $\sigma(\lambda, \mu)=\lambda a+\mu(1-a)$ for all $\lambda, \mu \in \mathbb{C}$. Note that $(0,0) \leq(1,0) \leq$ $(1,1)$ and thus $0 \leq a \leq 1$. Let $C^{*}(a)$ be the $C^{*}$-subalgebra of $\mathscr{A}$ generated by $a$. Then $C^{*}(a)$ is commutative since $a$ is positive (and thus normal). Given a MIU-map $\omega: C^{*}(a) \rightarrow \mathbb{C}$ we have $\omega(a) \in[0,1]$ since $0 \leq a \leq 1$. Thus $\omega \mapsto \omega(a)$ gives a map $j: \Sigma C^{*}(a) \rightarrow[0,1]$, where $\Sigma C^{*}(a)$ is the spectrum of $C^{*}(a)$, that is, $\Sigma C^{*}(a)$ is the set of MIU-maps from $C^{*}(a)$ to $\mathbb{C}$ with the topology of pointwise convergence. (By the way, the image of $j$ is the spectrum of the element $a$.) The map $j$ is continuous since the topology on $\Sigma C^{*}(a)$ is induced by the product topology on $\mathbb{C}^{C^{*}(a)}$. Thus the assignment $h \mapsto h \circ j$ gives a MIU-map $C j: C[0,1] \rightarrow C \Sigma C^{*}(a)$. By Gelfand's representation theorem there is a MIU-isomorphism

$$
\gamma: C^{*}(a) \longrightarrow C \Sigma C^{*}(a)
$$

given by $\gamma(b)(\omega)=\omega(b)$ for all $b \in C^{*}(a)$ and $\omega \in \Sigma C^{*}(a)$. Now, define

$$
\bar{\sigma}:=\gamma^{-1} \circ C j: C[0,1] \longrightarrow \mathbb{C}^{*}(a) \hookrightarrow \mathscr{A} .
$$

(In the language of the functional calculus, $\bar{\sigma}$ maps $f$ to $f(a)$.) We claim that $\bar{\sigma} \circ \rho=\sigma$. It suffices to 
show that $C j \circ \rho \equiv \gamma \circ \bar{\sigma} \circ \rho=\gamma \circ \sigma$. Let $\lambda, \mu \in \mathbb{C}$ and $\omega \in \Sigma C^{*}(a)$ be given. We have

$$
\begin{aligned}
(C j \circ \rho)(\lambda, \mu)(\omega) & =(C j)(\rho(\lambda, \mu))(\omega) & & \\
& =\rho(\lambda, \mu)(j(\omega)) & & \text { by def. of } C j \\
& =\lambda j(\omega)+\mu(1-j(\omega)) & & \text { by def. of } \rho \\
& =\lambda \omega(a)+\mu(1-\omega(a)) & & \text { by def. of } j \\
& =\omega(\lambda a+\mu(1-a)) & & \text { as } \omega \text { is a MIU-map } \\
& =\omega(\sigma(\lambda, \mu)) & & \text { by choice of } a \\
& =\gamma(\sigma(\lambda, \mu))(\omega) . & & \text { by def. of } \gamma \\
& =(\gamma \circ \sigma)(\lambda, \mu)(\omega) . & &
\end{aligned}
$$

It remains to be shown that $\bar{\sigma}$ is the only MIU-map $\tau: C[0,1] \rightarrow \mathscr{A}$ such that $U \tau \circ \rho=\sigma$. Let $\tau$ be such a map; we prove that $\tau=\bar{\sigma}$. By assumption $\tau$ and $\bar{\sigma}$ agree on the elements $f \in C[0,1]$ of the form

$$
f(x)=\lambda x+\mu(1-x) .
$$

In particular, $\bar{\sigma}$ and $\tau$ agree on the map $h:[0,1] \rightarrow \mathbb{C}$ given by $h(x)=x$.

Now, since $\bar{\sigma}$ and $\tau$ are MIU-maps and $h$ generates the $C^{*}$-algebra $C[0,1]$ (this is Weierstrass's theorem), it follows that $\bar{\sigma}=\tau$.

Example 3. The image of $\mathbb{C}^{3}$ under $F$ will not be commutative, or more formally:

If $\rho: \mathbb{C}^{3} \longrightarrow U \mathscr{B}$ is an initial map from $\mathbb{C}^{3}$ to $U$, then $\mathscr{B}$ is not commutative.

Suppose that $\mathscr{B}$ is commutative towards contradiction. Let $\mathscr{A}$ be a $C^{*}$-algebra in which there are positive $a_{1}, a_{2}, a_{3}$ such that $a_{1} a_{2} \neq a_{2} a_{1}$ and $a_{1}+a_{2}+a_{3}=1$.

(For example, we can take $\mathscr{A}$ to be the set of linear operators on $\mathbb{C}^{2}$ and let

$$
a_{1}:=1 / 2 P_{1} \quad a_{2}:=1 / 2 P_{+} \quad a_{3}:=I-1 / 2 P_{1}-1 / 2 P_{+}
$$

where $P_{1}$ denotes the orthogonal projection onto $\{(0, x): x \in \mathbb{C}\}$ and $P_{+}$is the orthogonal projection onto $\{(x, x): x \in \mathbb{C}\}$.)

Define $f: \mathbb{C}^{3} \rightarrow \mathscr{A}$ by, for all $\lambda_{1}, \lambda_{2}, \lambda_{3} \in \mathbb{C}$,

$$
f\left(\lambda_{1}, \lambda_{2}, \lambda_{3}\right)=\lambda_{1} a_{1}+\lambda_{2} a_{2}+\lambda_{3} a_{3}
$$

Then it is not hard to see that $f$ a PU-map. So as $\mathscr{B}$ is the initial arrow from $\mathbb{C}^{3}$ to $U$ there is a (unique) MIU-map $\bar{f}: \mathscr{B} \rightarrow \mathscr{A}$ such that $\bar{f} \circ \rho=f$. We have

$$
\begin{aligned}
a_{1} \cdot a_{2} & =f(1,0,0) \cdot f(0,1,0) \\
& =\bar{f}(\rho(1,0,0)) \cdot \bar{f}(\rho(0,1,0)) \\
& =\bar{f}(\rho(1,0,0) \cdot \rho(0,1,0)) \\
& =\bar{f}(\rho(0,1,0) \cdot \rho(1,0,0)) \quad \text { because } \mathscr{B} \text { is commutative } \\
& =\bar{f}(\rho(0,1,0)) \cdot \bar{f}(\rho(1,0,0)) \\
& =a_{2} \cdot a_{1} .
\end{aligned}
$$

This contradicts $a_{1} \cdot a_{2} \neq a_{2} \cdot a_{1}$. Hence $\mathscr{B}$ is not commutative. 
Remark 4. Before we prove that the embedding $\mathbf{C}_{\mathrm{MIU}}^{*} \rightarrow \mathbf{C}_{\mathrm{PU}}^{*}$ has a left adjoint $F$ (see Theorem 5 ) let us compare what we already know about $F$ with the commutative case. Let $\mathbf{C C}_{\mathrm{MIU}}^{*}$ denote the category of MIU-maps between commutative $C^{*}$-algebras and let $\mathbf{C C}_{\mathrm{PU}}^{*}$ denote the category of PU-maps between commutative $C^{*}$-algebras. From the work in [3] it follows that the embedding $\mathbf{C C}_{\mathrm{MIU}}^{*} \longrightarrow \mathbf{C C}_{\mathrm{PU}}^{*}$ has a left adjoint $F^{\prime}$ and moreover that $F^{\prime} \mathscr{A}=C$ Stat $\mathscr{A}$, where Stat $\mathscr{A}$ is the topological space of PU-maps from $\mathscr{A}$ to $\mathbb{C}$ with pointwise convergence and $C$ Stat $\mathscr{A}$ is the $C^{*}$-algebra of continuous functions from Stat $\mathscr{A}$ to $\mathbb{C}$.

Let $x \in[0,1]$. Then the assignment $(\lambda, \mu) \mapsto x \lambda+(1-x) \mu$ gives a PU-map $\bar{x}: \mathbb{C}^{2} \rightarrow \mathbb{C}$. It is not hard to see that $x \mapsto \bar{x}$ gives an isomorphism from $[0,1]$ to $\operatorname{Stat} \mathbb{C}^{2}$. Thus $F^{\prime} \mathbb{C}^{2} \cong C[0,1]$. Hence on $\mathbb{C}^{2}$ the functor $F$ and its commutative variant $F^{\prime}$ agree (see Example 2). However, on $\mathbb{C}^{3}$ the functors $F$ and $F^{\prime}$ differ. Indeed, $F^{\prime} \mathbb{C}^{3}$ is commutative while $F \mathbb{C}^{3}$ is not (see Example 3 .

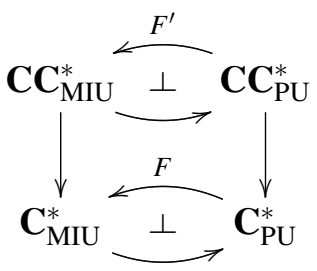

Roughly summarised: while in the diagram above the right adjoints commute with the vertical embeddings, the left adjoints do not.

Theorem 5. The embedding $U: \mathbf{C}_{\mathrm{MIU}}^{*} \longrightarrow \mathbf{C}_{\mathrm{PU}}^{*}$ has a left adjoint.

Proof. By Freyd's Adjoint Functor Theorem (see Theorem V.6.1 of [6]) and the fact that all limits can be formed using only products and equalisers (see Theorem V.2.1 and Exercise V.4.2 of [6]) it suffices to prove the following.

(i) The category $\mathbf{C}_{\mathrm{MIU}}^{*}$ has all small products and equalisers.

(ii) The functor $U: \mathbf{C}_{\mathrm{MIU}}^{*} \longrightarrow \mathbf{C}_{\mathrm{PU}}^{*}$ preserves small products and equalisers.

(iii) Solution Set Condition. For every $C^{*}$-algebra $\mathscr{A}$ there is a set $I$ and for each $i \in I$ a PU-map $f_{i}: \mathscr{A} \rightarrow \mathscr{A}_{i}$ such that for any PU-map $f: \mathscr{A} \rightarrow \mathscr{B}$ there is an $i \in I$ and a MIU-map $h: \mathscr{A}_{i} \rightarrow \mathscr{B}$ such that $h \circ f_{i}=f$.

Conditions (i) and (ii) can be verified with routine so we will spend only a few words on them (and leave the details to the reader). To see that Condition (iii) holds requires a little more ingenuity and so we will give the proof in detail.

(Conditions (i) and (ii)) Let us first think about small products in $\mathbf{C}_{\mathrm{MIU}}^{*}$ and $\mathbf{C}_{\mathrm{PU}}^{*}$.

Let $I$ be a set, and for each $i \in I$ let $\mathscr{A}_{i}$ be a $C^{*}$-algebra.

It is not hard to see that cartesian product $\prod_{i \in I} \mathscr{A}_{i}$ is a $*$-algebra when endowed with coordinate-wise operations (and it is in fact the product of the $\mathscr{A}_{i}$ in the category of $*$-algebras with MIU-maps, and with PU-maps).

However, $\prod_{i \in I} \mathscr{A}_{i}$ cannot be the product of the $\mathscr{A}_{i}$ as $C^{*}$-algebras: there is not even a $C^{*}$-norm on $\prod_{i \in I} \mathscr{A}_{i}$ unless $\mathscr{A}_{i}$ is trivial for all but finitely many $i \in I$. Indeed, if $\|-\|$ were a $C^{*}$-norm on $\prod_{i \in I} \mathscr{A}_{i}$, then we must have $\|\sigma(i)\| \leq\|\sigma\|$ for all $\sigma \in \prod_{i \in I} \mathscr{A}_{i}$ and $i \in I$, and so for any sequence $i_{0}, i_{1}, \ldots$ of distinct elements of $I$ for which $\mathscr{A}_{i_{0}}, \mathscr{A}_{i_{1}}, \ldots$ are non-trivial, and for every $\sigma \in \prod_{i \in I} \mathscr{A}_{i}$ with $\sigma\left(i_{n}\right)=n \cdot 1$ for all $n$, we have $n=\left\|\sigma\left(i_{n}\right)\right\| \leq\|\sigma\|$ for all $n$, so $\|\sigma\|=\infty$, which is not allowed.

Nevertheless, the $*$-subalgebra of $\prod_{i \in I} \mathscr{A}_{i}$ given by

$$
\bigoplus_{i \in I} \mathscr{A}_{i}:=\left\{\sigma \in \prod_{i \in I} \mathscr{A}_{i}: \sup _{i \in I}\|\sigma(i)\|<+\infty\right\}
$$


is a $C^{*}$-algebra with norm given by, for $\sigma \in \bigoplus_{i \in I} \mathscr{A}_{i}$,

$$
\|\sigma\|=\sup _{i \in I}\|\sigma(i)\| .
$$

We claim that $\bigoplus_{i \in I} \mathscr{A}_{i}$ is the product of the $\mathscr{A}_{i}$ in $\mathbf{C}_{\mathrm{PU}}^{*}$ (and in $\mathbf{C}_{\mathrm{MIU}}^{*}$ ).

Let $\mathscr{C}$ be a $C^{*}$-algebra, and for each $i \in I$, let $f_{i}: \mathscr{C} \rightarrow \mathscr{A}_{i}$ be a PU-map. We must show that there is a unique PU-map $f: \mathscr{C} \rightarrow \bigoplus_{i \in I} \mathscr{A}_{i}$ such that $\pi_{i} \circ f=f_{i}$ for all $i \in I$ where $\pi_{i}: \bigoplus_{j \in I} \mathscr{A}_{j} \rightarrow \mathscr{A}_{i}$ is the $i$-th projection. It is clear that there is at most one such $f$, and it would satisfy for all $i \in I$, and $c \in \mathscr{C}$, $f(c)(i)=f_{i}(c)$.

To see that such map $f$ exists is easy if we are able to prove that, for all $c \in \mathscr{C}$,

$$
\sup _{i \in I}\left\|f_{i}(c)\right\|<+\infty .
$$

Let $i \in I$ be given. We claim that that $\left\|f_{i}(c)\right\| \leq\|c\|$ for any positive $c \in \mathscr{C}$. Indeed, we have $c \leq\|c\| \cdot 1$, and thus $f_{i}(c) \leq\|c\| \cdot f(1)=\|c\| \cdot 1$, and so $\left\|f_{i}(c)\right\| \leq\|c\|$. It follows that $\left\|f_{i}(c)\right\| \leq 4 \cdot\|c\|$ for any $c \in \mathscr{A}$ by writing $c=c_{1}-c_{2}+i c_{3}-i c_{4}$ where $c_{1}, c_{2}, c_{3}, c_{4} \in \mathscr{C}$ are all positive. (We even have $\|f(c)\| \leq\|c\|$ for all $c \in \mathscr{C}$, but this requires a bit more effor 1 ) Thus, we have $\sup _{i \in I}\left\|f_{i}(c)\right\| \leq 4\|c\|<+\infty$. Hence Statement (1) holds.

Thus $\bigoplus_{i \in I} \mathscr{A}_{i}$ is the product of the $\mathscr{A}_{i}$ in $\mathbf{C}_{\mathrm{PU}}^{*}$. It is easy to see that $\bigoplus_{i \in I} \mathscr{A}_{i}$ is the product of the $\mathscr{A}_{i}$ in $\mathbf{C}_{\mathrm{MIU}}^{*}$ as well. Hence $\mathbf{C}_{\mathrm{MIU}}^{*}$ has all small products (as does $\mathbf{C}_{\mathrm{PU}}^{*}$ ) and $U: \mathbf{C}_{\mathrm{MIU}}^{*} \longrightarrow \mathbf{C}_{\mathrm{PU}}^{*}$ preserves small products.

Let us think about equalisers in $\mathbf{C}_{\mathrm{MIU}}^{*}$ and $\mathbf{C}_{\mathrm{PU}}^{*}$. Let $\mathscr{A}$ and $\mathscr{B}$ be $C^{*}$-algebras and let $f, g: \mathscr{A} \rightarrow \mathscr{B}$ be MIU-maps. We must prove that $f$ and $g$ have an equaliser $e: \mathscr{E} \rightarrow \mathscr{A}$ in $\mathbf{C}_{\mathrm{MIU}}^{*}$, and that $e$ is the equaliser of $f$ and $g$ in $\mathbf{C}_{\mathrm{PU}}^{*}$ as well.

Since $f$ and $g$ are MIU-maps (and hence continuous), it is not hard to see that

$$
\mathscr{E}:=\{a \in \mathscr{A}: f(a)=g(a)\}
$$

is a $C^{*}$-subalgebra of $\mathscr{A}$. We claim that the inclusion $e: \mathscr{E} \rightarrow \mathscr{A}$ is the equaliser of $f, g$ in $\mathbf{C}_{\mathrm{PU}}^{*}$. Let $\mathscr{D}$ be a $C^{*}$-algebra and let $d: \mathscr{D} \rightarrow \mathscr{A}$ be a PU-map such that $f \circ d=g \circ d$. We must show that there is a unique PU-map $h: \mathscr{D} \rightarrow \mathscr{E}$ such that $d=e \circ h$. Note that $d$ maps $\mathscr{A}$ into $\mathscr{E}$. The map $h: \mathscr{D} \rightarrow \mathscr{E}$ is simply the restriction of $d: \mathscr{D} \rightarrow \mathscr{A}$ in the codomain. Hence $e$ is the equaliser of $f, g$ in $\mathbf{C}_{\mathrm{PU}}^{*}$.

Note that in the argument above $h$ is a PU-map since $d$ is a PU-map. If $d$ were a MIU-map, then $h$ would be a MIU-map too. Hence $e$ is the equaliser of $f, g$ in the category $\mathbf{C}_{\mathrm{MIU}}^{*}$ as well.

Hence $\mathbf{C}_{\mathrm{MIU}}^{*}$ has all equalisers and $U: \mathbf{C}_{\mathrm{MIU}}^{*} \longrightarrow \mathbf{C}_{\mathrm{PU}}^{*}$ preserves equalisers. Hence $\mathbf{C}_{\mathrm{MIU}}^{*}$ has all small limits and $U: \mathbf{C}_{\mathrm{MIU}}^{*} \longrightarrow \mathbf{C}_{\mathrm{PU}}^{*}$ preserves all small limits.

(Note that while we have seen that $\mathbf{C}_{\mathrm{PU}}^{*}$ has all small products, and it was easy to see that $\mathbf{C}_{\mathrm{MIU}}^{*}$ has all equalisers, it is not clear whether $\mathbf{C}_{\mathrm{PU}}^{*}$ has all equalisers. Indeed, if $f, g: \mathscr{A} \rightarrow \mathscr{B}$ are PU-maps, then the set $\{a \in \mathscr{A}: f(a)=g(a)\}$ need not be a $C^{*}$-subalgebra of $\mathscr{A}$.)

(Condition (iii)). Let $\mathscr{A}$ be a $C^{*}$-algebra. We must find a set $I$ and for each $i \in I$ a PU-map $f_{i}: \mathscr{A} \rightarrow \mathscr{A}_{i}$ such that for every PU-map $f: \mathscr{A} \rightarrow \mathscr{B}$ there is a (not necessarily unique) $i \in I$ and $h: \mathscr{A}_{i} \rightarrow \mathscr{B}$ such that $f=h \circ f_{i}$.

Note that if $f: \mathscr{A} \rightarrow \mathscr{B}$ is a PU-map, then the range of the PU-map $f$ need not be a $C^{*}$-subalgebra of $\mathscr{B}$. (If the range of PU-maps would have been $C^{*}$-algebras, then we could have taken $I$ to be the set of all ideals of $\mathscr{A}$, and $f_{J}: \mathscr{A} \rightarrow \mathscr{A} / J$ to be the quotient map for any ideal $J$ of $\mathscr{A}$.)

\footnotetext{
${ }^{1}$ See Corollary 1 of $[7]$.
} 
Nevertheless, given a PU-map $f: \mathscr{A} \rightarrow \mathscr{B}$ there is a smallest $C^{*}$-subalgebra, say $\mathscr{B}^{\prime}$, of $\mathscr{B}$ that contains the range of $f$. We claim that $\# \mathscr{B}^{\prime} \leq \#\left(\mathscr{A}^{\mathbb{N}}\right)$ where $\# \mathscr{B}^{\prime}$ is the cardinality of $\mathscr{B}^{\prime}$ and $\#\left(\mathscr{A}^{\mathbb{N}}\right)$ is the cardinality of $\mathscr{A}^{\mathbb{N}}$

If we can find proof for our claim, the rest is easy. Indeed, to begin note that the collection of all $C^{*}$ algebras is not a small set. However, given a set $U$, the collection of all $C^{*}$-algebras $\mathscr{C}$ whose elements come from $U$ (so $\mathscr{C} \subseteq U$ ) is a small set. Now, let $\kappa:=\#\left(\mathscr{A}^{\mathbb{N}}\right)$ be the cardinality of $\mathscr{A}^{\mathbb{N}}$ (so $\kappa$ is itself a set) and take

$$
I:=\left\{(\mathscr{C}, c): \mathscr{C} \text { is a } C^{*} \text {-algebra on a subset of } \kappa \text { and } c: \mathscr{A} \rightarrow \mathscr{C} \text { is a PU-map }\right\}
$$

Since the collection of $C^{*}$-algebras $\mathscr{C}$ with $\mathscr{C} \subseteq \kappa$ is small, and since the collection of PU-maps from $\mathscr{A}$ to $\mathscr{C}$ is small for any $C^{*}$-algebra $\mathscr{C}$, it follows that $I$ is small.

For each $i \in I$ with $i \equiv(\mathscr{C}, c)$ define $\mathscr{A}_{i}:=\mathscr{C}$ and $f_{i}:=c$.

Let $f: \mathscr{A} \rightarrow \mathscr{B}$ be a PU-map. We must find $i \in I$ and a MIU-map $h: \mathscr{A}_{i} \rightarrow \mathscr{B}$ such that $h \circ f_{i}=f$. Let $\mathscr{B}^{\prime}$ be the smallest $C^{*}$-subalgebra that contains the range of $f$. By our claim we have $\# \mathscr{B}^{\prime} \leq \#\left(\mathscr{A}^{\mathbb{N}}\right) \equiv$ $\kappa$. By renaming the elements of $\mathscr{B}^{\prime}$ we can find a $C^{*}$-algebra $\mathscr{C}$ isomorphic to $\mathscr{B}^{\prime}$ whose elements come from $\kappa$. Let $\varphi: \mathscr{C} \rightarrow \mathscr{B}^{\prime}$ be the isomorphism.

Note that $c:=\varphi^{-1} \circ f: \mathscr{A} \rightarrow \mathscr{C}$ is a PU-map. So we have $i:=(\mathscr{C}, c) \in I$. Further, the inclusion $e: \mathscr{B}^{\prime} \rightarrow \mathscr{B}$ is a MIU-map, as is $\varphi$. So we have:

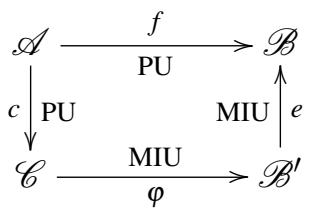

Now, $h:=e \circ \varphi: \mathscr{C} \rightarrow \mathscr{B}$ is a MIU-map with $f=h \circ f_{i}$. Hence Cond.(iii) holds.

Let us proof our claim. Let $\mathscr{A}$ and $\mathscr{B}$ be $C^{*}$-algebras and let $f: \mathscr{A} \rightarrow \mathscr{B}$ be a PU-map. Let $\mathscr{B}^{\prime}$ be the smallest $C^{*}$-subalgebra that contains the range of $f$.

We must show that $\# \mathscr{B}^{\prime} \leq \#\left(\mathscr{A}^{\mathbb{N}}\right)$.

Let us first take care of pathological case. Note that if $\mathscr{A}$ is trivial, i.e. $\mathscr{A}=\{0\}$, then $\mathscr{B}^{\prime}=\{0\}$, so $\#\left(\mathscr{A}^{\mathbb{N}}\right)=1=\# \mathscr{B}^{\prime}$. Now, let us assume that $\mathscr{A}$ is not trivial. Then we have an injection $\mathbb{C} \rightarrow \mathscr{A}$ given

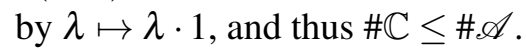

The trick to prove $\# \mathscr{B}^{\prime} \leq \#\left(\mathscr{A}^{\mathbb{N}}\right)$ is to find a more explicit description of $\mathscr{B}^{\prime}$. Let $T$ be the set of terms formed using a unary operation $(-)^{*}$ (involution) and two binary operations, $\cdot$ (multiplication) and + (addition), starting from the elements of $\mathscr{A}$. Let $f_{T}: T \longrightarrow \mathscr{B}^{\prime}$ be the map (recursively) given by, for $a \in \mathscr{A}$, and $s, t \in T$,

$$
\begin{aligned}
f_{T}(a) & =f(a) \\
f_{T}\left(s^{*}\right) & =\left(f_{T}(s)\right)^{*} \\
f_{T}(s \cdot t) & =f_{T}(s) \cdot f_{T}(t) \\
f_{T}(s+t) & =f_{T}(s)+f_{T}(t) .
\end{aligned}
$$

\footnotetext{
${ }^{2}$ Although it has no bearing on the validity of the proof one might wonder if the simpler statement $\# \mathscr{B}^{\prime} \leq \# \mathscr{A}$ holds as well. Indeed, if $\# \mathscr{A}=\# \mathbb{C}$ or $\# \mathscr{A}=\#\left(2^{X}\right)$ for some infinite set $X$, then we have $\# \mathscr{A}=\#\left(\mathscr{A}^{\mathbb{N}}\right)$, and so \#舟 $\leq \# \mathscr{A}$. However, not every uncountable set is of the form $2^{X}$ for some infinite set $X$, and in fact, if $\# \mathscr{A}=\mathfrak{\aleph}_{\omega}$, then $\#\left(\mathscr{A}^{\mathbb{N}}\right)>\# \mathscr{A}$ by Corollary 3.9 .6 of [2]
} 
Note that the range of $f_{B}$, let us call it $\operatorname{Ran} f_{B}$, is a $*$-subalgebra of $\mathscr{B}^{\prime}$. We will prove that \#Ran $f_{B} \leq \# \mathscr{A}$. Since $f_{B}$ is a surjection of $T$ onto $\operatorname{Ran} f_{B}$ it suffices to prove that $\# T \leq \# \mathscr{A}$. In fact, we will show that $\# T=\# \mathscr{A}$.

First note that $\mathscr{A}$ is infinite, and $\mathscr{A} \subseteq T$, so $T$ is infinite as well. To prove that $\# T=\# \mathscr{A}$ we write the elements of $T$ as words (with the use of brackets). Indeed, with $Q:=\mathscr{A} \cup\{$ “.", "“+”, “*”, ")", “(”\} there is an obvious injection from $T$ into the set $Q^{*}$ of words over $Q$. Since $\mathscr{A}$ is infinite, and $Q \backslash \mathscr{A}$ is finite we have $\# Q=\# \mathscr{A}$ by Hilbert's hotel. Recall that $Q^{*}=\bigcup_{n=0}^{\infty} Q^{n}$. Since $Q$ is infinite, we also have $\#(\mathbb{N} \times Q)=\# Q$ and even $\#(Q \times Q)=\# Q$ (see Theorem 3.7.7 of [2]), so \#Q=\#( $\left.Q^{n}\right)$ for all $n>0$. It follows that

$$
\begin{aligned}
\#\left(Q^{*}\right) & =\#\left(\bigcup_{n=0}^{\infty} Q^{n}\right) \\
& =\#\left(1+\bigcup_{n=1}^{\infty} Q\right) \\
& =\#(1+\mathbb{N} \times Q) \\
& =\# Q .
\end{aligned}
$$

Since there is an injection from $T$ to $Q^{*}$ we have $\# \mathscr{A} \leq \# T \leq \#\left(Q^{*}\right)=\# Q=\# \mathscr{A}$ and so $\# T=\# \mathscr{A}$. Hence \#Ran $f_{B} \leq \# \mathscr{A}$.

Since $\operatorname{Ran} f_{B}$ is a $*$-algebra that contains $\operatorname{Ran} f$, the closure $\overline{\operatorname{Ran} f_{B}}$ of $\operatorname{Ran} f_{B}$ with respect to the norm on $\mathscr{B}^{\prime}$ is a $C^{*}$-algebra that contains $\operatorname{Ran} f$. As $\mathscr{B}^{\prime}$ is the smallest $C^{*}$-subalgebra that contains $\operatorname{Ran} f$, we see that $\mathscr{B}^{\prime}=\overline{\operatorname{Ran} f_{B}}$.

Let $S$ be the set of all Cauchy sequences in $\operatorname{Ran} f_{B}$. As every point in $\mathscr{B}^{\prime}$ is the limit of a Cauchy sequence in $\operatorname{Ran} f_{B}$, we get $\# \mathscr{B}^{\prime} \leq \# S$. Thus:

$$
\begin{array}{rlrl}
\# \mathscr{B}^{\prime} & \leq \# S & \\
& \leq \#\left(\operatorname{Ran} f_{B}\right)^{\mathbb{N}} & & \text { as } S \subseteq\left(\operatorname{Ran} f_{B}\right)^{\mathbb{N}} \\
& \leq \#\left(\mathscr{A}^{\mathbb{N}}\right) & & \text { as } \# \operatorname{Ran} f_{B} \leq \# \mathscr{A} .
\end{array}
$$

Thus we have proven our claim.

Hence Conditions (i) (iii) hold and $U: \mathbf{C}_{\mathrm{MIU}}^{*} \longrightarrow \mathbf{C}_{\mathrm{PU}}^{*}$ has a left adjoint.

We have seen that $U: \mathbf{C}_{\mathrm{MIU}}^{*} \longrightarrow \mathbf{C}_{\mathrm{PU}}^{*}$ has a left adjoint $F: \mathbf{C}_{\mathrm{PU}}^{*} \longrightarrow \mathbf{C}_{\mathrm{MIU}}^{*}$. This adjunction gives a comonad $F U$ on $\mathbf{C}_{\mathrm{MIU}}^{*}$, which in turns gives us two categories: the Eilenberg-Moore category $\mathscr{E} \mathscr{M}(F U)$ of $F U$-coalgebras and the Kleisli category $\mathscr{K} \ell(F U)$. We claim that $\mathbf{C}_{\mathrm{PU}}^{*}$ is isomorphic to $\mathscr{K} \ell(F U)$ since $\mathbf{C}_{\mathrm{MIU}}^{*}$ is a subcategory of $\mathbf{C}_{\mathrm{PU}}^{*}$ with the same objects.

This is a special case of a more general phenomenon which we discuss in the next section (in terms of monads instead of comonads), see Theorem 9

\section{Kleislian Adjunctions}

Beck's Theorem (see [6], VI.7) gives a criterion for when an adjunction $F \dashv U$ "is" an adjunction between C and $\mathscr{E} \mathscr{M}(U F)$. We give a similar (but easier) criterion for when an adjunction "is" an adjunction between $\mathbf{C}$ and $\mathscr{K} \ell(U F)$. The criterion is not new; e.g., it is mentioned in [5] (paragraph 8.6) without proof or reference, and it can be seen as a consequence of Exercise VI.5.2 of [6] (if one realises that an equivalence which is bijective on objects is an isomorphism). Proofs can be found in the appendix. 
Notation 6. Let $F: \mathbf{C} \longrightarrow \mathbf{D}$ be a functor with right adjoint $U$. Denote the unit of the adjunction by $\eta: \mathrm{id}_{\mathbf{D}} \rightarrow U F$, and the counit by $\varepsilon: F U \rightarrow \mathrm{id}_{\mathbf{C}}$.

Recall that $U F$ is a monad with unit $\eta$ and as multiplication, for $C$ from $\mathbf{C}$,

$$
\mu_{C}:=U \varepsilon_{F C}: U F U F C \longrightarrow U F C
$$

Let $\mathscr{K} \ell(U F)$ be the Kleisli category of the monad UF. So $\mathscr{K} \ell(U F)$ has the same objects as $\mathbf{C}$, and the morphisms in $\mathscr{K} \ell(U F)$ from $C_{1}$ to $C_{2}$ are the morphism in $\mathbf{C}$ from $C_{1}$ to $U F C_{2}$. Given $C$ from $\mathbf{C}$ the identity in $\mathscr{K} \ell(U F)$ on $C$ is $\eta_{C}$. If $C_{1}, C_{2}, C_{3}, f: C_{1} \rightarrow C_{2}, g: C_{2} \rightarrow C_{3}$ from $\mathbf{C}$ are given, $g$ after $f$ in $\mathscr{K} \ell(U F)$ is

$$
g \odot f:=\mu_{C_{3}} \circ U F g \circ f .
$$

Let $V: \mathbf{C} \longrightarrow \mathscr{K} \ell(U F)$ be given by, for $f: C_{1} \longrightarrow C_{2}$ from $\mathbf{C}$,

$$
V f:=\eta_{C_{2}} \circ f: \quad C_{1} \longrightarrow U F C_{2} .
$$

Let $G: \mathscr{K} \ell(U F) \longrightarrow \mathbf{C}$ be given by, for $f: C_{1} \longrightarrow U F C_{2}$ from $\mathbf{C}$,

$$
G f:=\mu_{C_{2}} \circ U F f: \quad U F C_{1} \longrightarrow U F C_{2} .
$$

The following is Exercise VI.5.1 of [6].

Lemma 7. Let $F: \mathbf{C} \longrightarrow \mathbf{D}$ be a functor with a right adjoint $U$.

Then there is a unique functor $L: \mathscr{K} \ell(U F) \longrightarrow \mathbf{D}$ (called the comparison functor) such that $U \circ L=G$ and $L \circ V=F$ (see Notation 6).

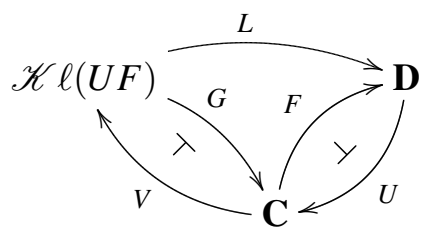

Definition 8. Let $\mathbf{C}$ and $\mathbf{D}$ be categories.

(i) A functor $F: \mathbf{C} \longrightarrow \mathbf{D}$ is called Kleislian when it has a right adjoint $U: \mathbf{D} \rightarrow \mathbf{C}$, and the functor $L: \mathscr{K} \ell(U F) \longrightarrow \mathbf{D}$ from Lemma 7 is an isomorphism.

(ii) We say that $\mathbf{D}$ is Kleislian over $\mathbf{C}$ when there is a Kleislian functor $F: \mathbf{C} \longrightarrow \mathbf{D}$.

Theorem 9. Let $F: \mathbf{C} \longrightarrow \mathbf{D}$ be a functor with a right adjoint $U$.

The following are equivalent.

(i) F is Kleislian (see Definition 8).

(ii) $F$ is bijective on objects (i.e. for every object $D$ from $\mathbf{D}$ there is a unique object $C$ from $\mathbf{C}$ such that $F C=D$ ).

Corollary 10. The embedding $U^{\mathrm{op}}:\left(\mathbf{C}_{\mathrm{MIU}}^{*}\right)^{\mathrm{op}} \longrightarrow\left(\mathbf{C}_{\mathrm{PU}}^{*}\right)^{\mathrm{op}}$ is Kleislian (see Def. 8).

Proof. By Theorem 9 we must show that $U^{\text {op }}$ has a left adjoint and is bijective on objects. Since the embedding $U: \mathbf{C}_{\mathrm{MIU}}^{*} \rightarrow \mathbf{C}_{\mathrm{PU}}^{*}$ has a left adjoint $F: \mathbf{C}_{\mathrm{PU}}^{*} \rightarrow \mathbf{C}_{\mathrm{MIU}}^{*}$ it follows that $F^{\mathrm{op}}:\left(\mathbf{C}_{\mathrm{PU}}^{*}\right)^{\mathrm{op}} \rightarrow\left(\mathbf{C}_{\mathrm{MIU}}^{*}\right)^{\text {op }}$ is the right adjoint of $U^{\mathrm{op}}$. Thus $U^{\mathrm{op}}$ has a left adjoint. Further, as $\mathbf{C}_{\mathrm{MIU}}^{*}$ and $\mathbf{C}_{\mathrm{PU}}^{*}$ have the same objects, $U$ is bijective on objects, and so is $U^{\text {op }}$. Hence $U^{\text {op }}$ is Kleislian. 
In summary, the embedding $U: \mathbf{C}_{\mathrm{MIU}}^{*} \longrightarrow \mathbf{C}_{\mathrm{PU}}^{*}$ has a left adjoint $F\left(\right.$ and so $F^{\mathrm{op}}:\left(\mathbf{C}_{\mathrm{MIU}}^{*}\right)^{\text {op }} \rightarrow\left(\mathbf{C}_{\mathrm{PU}}^{*}\right)^{\text {op }}$ is right adjoint to $\left.U^{\mathrm{op}}\right)$, and the unique functor from the Kleisli category $\mathscr{K} \ell(F U)$ of the monad $F U$ on $\left(\mathbf{C}_{\mathrm{MIU}}^{*}\right)^{\text {op }}$ to $\left(\mathbf{C}_{\mathrm{PU}}^{*}\right)^{\text {op }}$ that makes the two triangles in the diagram below on the left commute is an isomorphism.
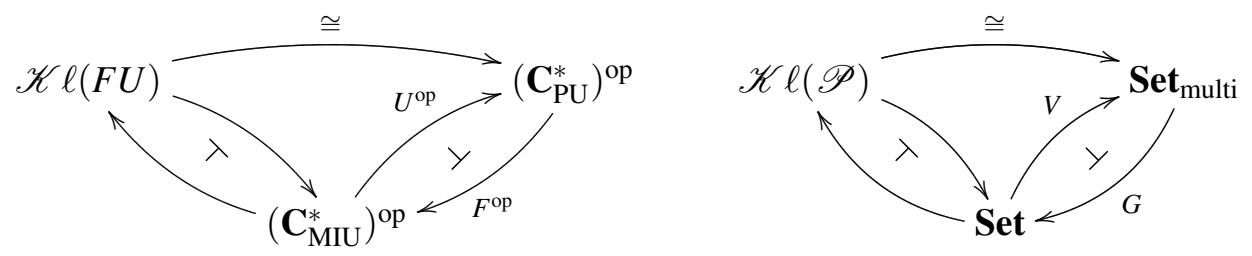

For the category $\mathbf{S e t}_{\text {multi }}$ of multimaps between sets used in the introduction to describe the semantics of non-deterministic programs the situation is the same, see the diagram above to the right.

(The functor $V$ is the obvious embedding. The right adjoint $G$ of $V$ sends a multimap $f$ from $X$ to $Y$ to the function $G f: \mathscr{P}(X) \rightarrow \mathscr{P}(Y)$ that assigns to a subset $A \in \mathscr{P}(X)$ the image of $A$ under $f$. Note that $G V=\mathscr{P}$.)

\section{Discussion}

\subsection{Variations}

Example 11 (Subunital maps). Let $\mathbf{C}_{\mathrm{PsU}}^{*}$ be the category of $C^{*}$-algebras and the positive linear maps $f$ between them that are subunitial, i.e. $f(1) \leq 1$. The morphisms of $\mathbf{C}_{\mathrm{PsU}}^{*}$ are called PsU-maps.

It is not hard to see that the products in $\mathbf{C}_{\mathrm{PsU}}^{*}$ are the same as in $\mathbf{C}_{\mathrm{MIU}}^{*}$, and that the equaliser in $\mathbf{C}_{\mathrm{MIU}}^{*}$ of a pair $f, g$ of MIU-maps is the equaliser of $f, g$ in $\mathbf{C}_{\mathrm{PsU}}^{*}$ as well. Thus the embedding $U: \mathbf{C}_{\mathrm{MIU}}^{*} \longrightarrow \mathbf{C}_{\mathrm{PsU}}^{*}$ preserves limits. Using the same argument as in Theorem 5 but with "PU-map" replaced by "PsU-map" one can show that $U$ satisfies the Solution Set Condition. Hence $U$ has a left adjoint by Freyd's Adjoint Function Theorem, say $F: \mathbf{C}_{\mathrm{PsU}}^{*} \longrightarrow \mathbf{C}_{\mathrm{MIU}}^{*}$.

Since $\mathbf{C}_{\mathrm{PsU}}^{*}$ has the same objects as $\mathbf{C}_{\mathrm{MIU}}^{*}$ (namely the $C^{*}$-algebras) the functor $U^{\mathrm{op}}:\left(\mathbf{C}_{\mathrm{MIU}}^{*}\right)^{\mathrm{op}} \longrightarrow$ $\left(\mathbf{C}_{\mathrm{PsU}}^{*}\right)^{\text {op }}$ is bijective on objects and thus Kleislian (by Th. 9).

Hence $\left(\mathbf{C}_{\mathrm{PsU}}^{*}\right)^{\text {op }}$ is Kleislian over $\left(\mathbf{C}_{\mathrm{MIU}}^{*}\right)^{\mathrm{op}}$.

Example 12 (Bounded linear maps). Let $\mathbf{C}_{\mathrm{P}}^{*}$ be the category of positive bounded linear maps between $C^{*}$-algebras. We will show that $\left(\mathbf{C}_{\mathrm{P}}^{*}\right)^{\text {op }}$ is not Kleislian over $\left(\mathbf{C}_{\mathrm{MIU}}^{*}\right)^{\text {op }}$. Indeed, if it were then $\left(\mathbf{C}_{\mathrm{P}}^{*}\right)^{\text {op }}$ would be cocomplete, but it is not: there is no $\omega$-fold product of $\mathbb{C}$ in $\mathbf{C}_{\mathrm{P}}^{*}$. To see this, suppose that there is a $\omega$-fold product $\mathscr{P}$ in $\mathbf{C}_{\mathrm{P}}^{*}$ with projections $\pi_{i}: \mathscr{P} \rightarrow \mathbb{C}$ for $i \in \omega$. Since $\pi_{i}$ is a bounded linear map for $i \in \omega$, it has finite operator norm, say $\left\|\pi_{i}\right\|$. By symmetry, $\left\|\pi_{i}\right\|=\left\|\pi_{j}\right\|$ for all $i, j \in \omega$. Write $K:=\left\|\pi_{0}\right\|=\left\|\pi_{1}\right\|=\left\|\pi_{2}\right\|=\cdots$. Define $f_{i}: \mathbb{C} \rightarrow \mathbb{C}$ by $f_{i}(z)=i z$ for all $z \in \mathbb{C}$ and $i \in \omega$. Then $f_{i}$ is a positive bounded linear map for each $i \in \omega$. Since $\mathscr{P}$ is the $\omega$-fold product of $\mathbb{C}$, there is a (unique positive) bounded linear map $f: \mathbb{C} \rightarrow \mathscr{P}$ such that $\pi_{i} \circ f=f_{i}$ for all $i \in \omega$. For each $N \in \omega$ we have

$$
N=\left\|f_{N}(1)\right\| \leq\left\|f_{N}\right\|=\left\|\pi_{N} \circ f\right\| \leq\left\|\pi_{N}\right\|\|f\|=K\|f\| .
$$

Thus $K\|f\|$ is greater than any number, which is absurd.

Example 13 (Completely positive maps). For clarity's sake we recall what it means for a linear map $f$ between $C^{*}$-algebras to be completely positive (see [8]). For this we need some notation. Given a $C^{*}$ algebra $\mathscr{A}$, and $n \in \mathbb{N}$ let $M_{n}(\mathscr{A})$ denote the set of $n \times n$-matrices with entries from $\mathscr{A}$. We leave it to the 
reader to check that $M_{n}(\mathscr{A})$ is a $*$-algebra with the obvious operations. In fact, it turns out that $M_{n}(\mathscr{A})$ is a $C^{*}$-algebra, but some care must be taken to define the norm on $M_{n}(\mathscr{A})$ as we will see below. Now, a linear map $f: \mathscr{A} \longrightarrow \mathscr{B}$ is called completely positive when $M_{n} f$ is positive for each $n \in \mathbb{N}$, where $M_{n} f: M_{n}(\mathscr{A}) \longrightarrow M_{n}(\mathscr{B})$ is the map obtained by applying $f$ to each entry of a matrix in $M_{n}(\mathscr{A})$. Of course, " $M_{n} f$ is positive" only makes sense once we know that $M_{n}(\mathscr{A})$ and $M_{n}(\mathscr{B})$ are $C^{*}$-algebras.

Let $\mathscr{A}$ be a $C^{*}$-algebra. We will put a $C^{*}$-norm on $M_{n}(\mathscr{A})$. Let $\mathscr{H}$ be a Hilbert space and let $\pi: \mathscr{A} \longrightarrow \mathscr{B}(\mathscr{H})$, be an isometric MIU-map. We get a norm $\|-\|_{\pi}$ on $M_{n}(\mathscr{A})$ given by for $A \in M_{n}(\mathscr{A})$,

$$
\|A\|_{\pi}=\left\|\xi\left(\left(M_{n} \pi\right)(A)\right)\right\|
$$

where $\xi\left(\left(M_{n} \pi\right)(A)\right): \mathscr{H}^{\oplus n} \rightarrow \mathscr{H}^{\oplus n}$ is the bounded linear map represented by the matrix $\left(M_{n} \pi\right)(A)$, and $\left\|\xi\left(\left(M_{n} \pi\right)(A)\right)\right\|$ is the operator norm of $\xi\left(\left(M_{n} \pi\right)(A)\right)$ in $\mathscr{B}\left(\mathscr{H}^{\oplus n}\right)$.

It is easy to see that $\|-\|_{\pi}$ satisfies the $C^{*}$-identity, $\left\|A^{*} A\right\|_{\pi}=\|A\|_{\pi}^{2}$ for all $A \in M_{n}(\mathscr{A})$. It is less obvious that $M_{n}(\mathscr{A})$ is complete with respect to $\|-\| \|_{\pi}$. To see this, first note that $\left\|A_{i j}\right\| \leq\|A\|_{\pi}$ for all $i, j$. So given a Cauchy sequence $A_{1}, A_{2}, \ldots$ in $M_{n}(\mathscr{A})$ we can form the entrywise limit $A$, that is, $A_{i j}=\lim _{m \rightarrow \infty} A_{i j}$. We leave it to the reader to check that $A_{i j}$ is the limit of $A_{1}, A_{2}, \ldots$, and thus $M_{n}(\mathscr{A})$ is complete with respect to $\|-\|_{\pi}$. Hence $M_{n}(\mathscr{A})$ is a $C^{*}$-algebra with norm $\|-\|_{\pi}$.

The $C^{*}$-norm $\|-\|_{\pi}$ does not depend on $\pi$. Indeed, let $\mathscr{H}_{1}$ and $\mathscr{H}_{2}$ be Hilbert spaces and let $\pi_{1}: \mathscr{A} \longrightarrow \mathscr{B}\left(\mathscr{H}_{1}\right)$ and $\pi_{2}: \mathscr{A} \longrightarrow \mathscr{B}\left(\mathscr{H}_{2}\right)$ be isometric MIU-maps; we will show that $\|-\|_{\pi_{1}}=\|-\|_{\pi_{2}}$. Recall that the norm $\|-\| \pi_{i}$ induces an order $\leq_{\pi_{i}}$ on $M_{n}(\mathscr{A})$ given by $0 \leq \pi_{i} A$ iff $\|A-\| A\left\|_{\pi_{i}}\right\|_{\pi_{i}} \leq\|A\|_{\pi_{i}}$ where $A \in M_{n}(\mathscr{A})$. Since $\|A\|_{\pi_{i}}^{2}=\inf \left\{\lambda \in[0, \infty): A^{*} A \leq_{\pi_{i}} \lambda\right\}$ for all $A \in M_{n}(\mathscr{A})$, to prove $\|-\|_{\pi_{1}}=$ $\|-\|_{\pi_{2}}$ it suffices to show that the orders $\leq_{\pi_{1}}$ and $\leq_{\pi_{2}}$ coincide. But this is easy when one recalls that $A \in M_{n}(\mathscr{A})$ is positive iff $A$ is of the form $B^{*} B$ for some $B \in M_{n}(\mathscr{A})$.

The completely positive linear maps that preserve the unit are called $C P U$-maps. Let $\mathbf{C}_{\mathrm{CPU}}^{*}$ be the category of CPU-maps between $C^{*}$-algebras. Since $M_{n}(f)$ is a MIU-map when $f$ is a MIU-map and a MIU-map is positive, we see that any MIU-map is completely positive. Thus $\mathbf{C}_{\text {MIU }}^{*}$ is a subcategory of $\mathbf{C}_{\mathrm{CPU}}^{*}$. We claim that $\left(\mathbf{C}_{\mathrm{CPU}}^{*}\right)^{\text {op }}$ is Kleislian over $\left(\mathbf{C}_{\mathrm{MIU}}^{*}\right)^{\text {op }}$.

Let us show that $U$ preserves limits. To show that $U$ preserves equalisers, let $f, g: \mathscr{A} \longrightarrow \mathscr{B}$ be MIUmaps. Then $\mathscr{E}:=\{x \in \mathscr{A}: f(x)=g(x)\}$ is a $C^{*}$-subalgebra of $\mathscr{A}$ and the embedding $e: \mathscr{E} \rightarrow \mathscr{A}$ is an isometric MIU-map. Then $e$ is the equalisers of $f, g$ in $\mathbf{C}_{\text {MIU }}^{*}$; we will show that $e$ is the equaliser of $f, g$ in $\mathbf{C}_{\mathrm{CPU}}^{*}$. Let $\mathscr{C}$ be a $C^{*}$-algebra, and let $c: \mathscr{C} \rightarrow \mathscr{A}$ be a CPU-map such that $f \circ c=g \circ c$ Let $d: \mathscr{C} \rightarrow \mathscr{E}$ be the restriction of $c$. It turns out we must prove that $d$ is completely positive. Let $n \in \mathbb{N}$ be given. We must show that $M_{n} d: M_{n} \mathscr{C} \rightarrow M_{n} \mathscr{E}$ is positive. Note that $M_{n} e$ is an injective MIU-map and thus an isometry. So in order to prove that $M_{n} d$ is positive it suffices to show that $M_{n} e \circ M_{n} d=M_{n}(e \circ d)=M_{n} c$ is positive, which it is since $c$ is completely positive. Thus $e$ is the equaliser of $f, g$ in $\mathbf{C}_{\mathrm{CPU}}^{*}$. Hence $U$ preservers equalisers.

To show that $U$ preserves products, let $I$ be a set and for each $i \in I$ let $\mathscr{A}_{i}$ be a $C^{*}$-algebra. We will show that $\bigoplus_{i \in I} \mathscr{A}_{i}$ is the product of the $\mathscr{A}_{i}$ in $\mathbf{C}_{\mathrm{CPU}}^{*}$. Let $\mathscr{C}$ be a $C^{*}$-algebra, and for each $i \in I$, let $f_{i}: \mathscr{C} \rightarrow \mathscr{A}_{i}$ be a CPU-map. As before, let $f: \mathscr{C} \rightarrow \bigoplus_{i \in I} A_{i}$ be the map given by $f(x)(i)=f_{i}(x)$ for all $i \in I$ and $x \in \mathscr{C}$. Leaving the details to the reader it turns out that it suffices to show that $f$ is completely positive. Let $n \in \mathbb{N}$ be given. We must prove that $M_{n} f: M_{n}(\mathscr{C}) \longrightarrow M_{n}\left(\bigoplus_{i \in I} \mathscr{A}_{i}\right)$ is positive. Let $\varphi: M_{n}\left(\bigoplus_{i \in I} \mathscr{A}_{i}\right) \longrightarrow \bigoplus_{i \in I} M_{n}\left(\mathscr{A}_{i}\right)$ be the unique MIU-map such that $\pi_{i} \circ \varphi=M_{n} \pi_{i}$ for all $i \in I$. Then $\varphi$ is a MIU-isomorphism and thus to prove that $M_{n} f$ is positive, it suffices to show that $\varphi \circ M_{n} f$ is positive. Let $i \in I$ be given. We must prove that $\pi_{i} \circ \varphi \circ M_{n} f$ is positive. But we have $\pi_{i} \circ \varphi \circ M_{n} f=M_{n} \pi_{i} \circ M_{n} f=M_{n}\left(\pi_{i} \circ f\right)=M_{n} f_{i}$, which is positive since $f$ is completely positive. Thus $\bigoplus_{i \in I} \mathscr{A}_{i}$ is the product of the $\mathscr{A}_{i}$ in $\mathbf{C}_{\mathrm{CPU}}^{*}$ and hence $U$ preserves limits. 
With the same argument as in Theorem 9 the functor $U$ satisfies the Solution Set Condition and thus $U$ has a left adjoint. It follows that $U^{\mathrm{op}}:\left(\mathbf{C}_{\mathrm{MIU}}^{*}\right)^{\mathrm{op}} \longrightarrow\left(\mathbf{C}_{\mathrm{CPU}}^{*}\right)^{\text {op }}$ is Kleislian.

Example 14 ( $W^{*}$-algebras). Let $\mathbf{W}_{\mathrm{NMIU}}^{*}$ be the category of von Neumann algebras (also called $W^{*}$ algebras) and the MIU-maps between them that are normal, i.e., preserve suprema of upwards directed sets of self-adjoint elements. Let $\mathbf{W}_{\mathrm{NPU}}^{*}$ be the category of von Neumann and normal PU-maps. Note that $\mathbf{W}_{\mathrm{NMIU}}^{*}$ is a subcategory of $\mathbf{W}_{\mathrm{NPU}}^{*}$. We will prove that $\left(\mathbf{W}_{\mathrm{NPU}}^{*}\right)^{\text {op }}$ is Kleislian over $\left(\mathbf{W}_{\mathrm{NMIU}}^{*}\right)^{\text {op }}$.

It suffices to show that $U$ has a left adjoint. Again we follow the lines of the proof of Theorem 5 Products and equalisers in $\mathbf{W}_{\mathrm{NMIU}}^{*}$ are the same as in $\mathbf{C}_{\mathrm{MIU}}^{*}$. It is not hard to see that the embedding $U: \mathbf{W}_{\mathrm{NMIU}}^{*} \longrightarrow \mathbf{W}_{\mathrm{NPU}}^{*}$ preserves limits. To see that $U$ satisfies the Solution Set Condition we use the same method as before: given a von Neumann algebra $\mathscr{A}$, find a suitable cardinal $\kappa$ such that the following is a solution set.

$$
\begin{array}{r}
I:=\{(\mathscr{C}, c): \mathscr{C} \text { is a von Neumann algebra on a subset of } \kappa \\
\text { and } c: \mathscr{A} \longrightarrow \mathscr{C} \text { is a normal PU-map }\},
\end{array}
$$

Only this time we take $\kappa=\#(\wp(\wp(\mathscr{A})))$ instead of $\kappa=\#\left(\mathscr{A}^{\mathbb{N}}\right)$. We leave the details to the reader, but it follows from the fact that given a subset $X$ of a von Neumann algebra $\mathscr{B}$ the smallest von Neumann subalgebra $\mathscr{B}^{\prime}$ that contains $X$ has cardinality at most $\#(\wp(\wp(X)))$. Indeed, if $\mathscr{H}$ is a Hilbert space such that $\mathscr{B} \subseteq \mathscr{B}(\mathscr{H})$ (perhaps after renaming the elements of $\mathscr{B}$ ), then $\mathscr{B}^{\prime}$ is the closure (in the weak operator topology on $\mathscr{B}(\mathscr{H})$ ) of the smallest $*$-subalgebra containing $X$. Thus any element of $\mathscr{B}^{\prime}$ is the limit of a filter - a special type of net, see paragraph 12 of [9] — of $*$-algebra terms over $X$, of which there are no more than $\#(\wp(\wp(X)))$.

By a similar reasoning one sees that the opposite $\left(\mathbf{W}_{\mathrm{NCPsU}}^{*}\right)^{\text {op }}$ of the category of normal completely positive subunital linear maps between von Neumann algebras is Kleislian over $\left(\mathbf{W}_{\mathrm{NMIU}}^{*}\right)^{\text {op }}$. The existence of the adjoint to the inclusion $\mathbf{W}_{\mathrm{NMIU}}^{*} \rightarrow \mathbf{W}_{\mathrm{NCPs}}^{*}$ is key in our construction of a model of Selinger and Valiron's quantum lambda calculus by von Neumann algebras, see [1].

\subsection{Concrete description}

In this note we have shown that the embedding $U: \mathbf{C}_{\mathrm{MIU}}^{*} \longrightarrow \mathbf{C}_{\mathrm{PU}}^{*}$ has a left adjoint $F$, but we miss a concrete description of $F \mathscr{A}$ for all but the simplest $C^{*}$-algebras $\mathscr{A}$. What constitutes a "concrete description" is perhaps a matter of taste or occasion, but let us pose that it should at least enable us to describe the Eilenberg-Moore category $\mathscr{E} \mathscr{M}(F U)$ of the comonad $F U$. More concretely, it should settle the following problem.

Problem 15. Writing BOUS for the category of positive linear maps that preserve the unit between Banach order unit spaces, determine whether $\mathscr{E} \mathscr{M}(F U) \cong$ BOUS.

(An order unit space is an ordered vector space $V$ over $\mathbb{R}$ with an element 1 , the order unit, such that for all $v \in V$ there is $\lambda \in[0, \infty)$ such that $-\lambda \cdot 1 \leq v \leq \lambda \cdot 1$. The smallest such $\lambda$ is denoted by $\|v\|$. See [4] for more details. If $v \mapsto\|v\|$ gives a complete norm, $V$ is called a Banach order unit space.)

\subsection{MIU versus PU}

A second "problem" is to give a physical description (if there is any) of what it means for a quantum program's semantics to be a MIU-map (and not just a PU-map). A step in this direction might be to define for a $C^{*}$-algebra $\mathscr{A}$, a PU-map $\varphi: \mathscr{A} \rightarrow \mathbb{C}$, and $a, b \in \mathscr{A}$ the quantity

$$
\operatorname{Cov}_{\varphi}(a, b):=\varphi\left(a^{*} b\right)-\varphi(a)^{*} \varphi(b)
$$


and interpret it as the covariance between the observables $a$ and $b$ in state $\varphi$ of the quantum system $\mathscr{A}$. Let $T: \mathscr{A} \longrightarrow \mathscr{B}$ be a PU-map between $C^{*}$-algebras (so perhaps $T$ is the semantics of a quantum program). Then it is not hard to verify that $T$ is a MIU-map if and only if $T$ preserves covariance, that is,

$$
\operatorname{Cov}_{\varphi}(T a, T b)=\operatorname{Cov}_{\varphi \circ T}(a, b) \quad \text { for all } a, b \in \mathscr{A} .
$$

\section{Acknowledgements}

Example 2 and Example 3 were suggested by Robert Furber. I'm grateful that Jianchao Wu and Sander Uijlen spotted several errors in a previous version of this text. Kenta Cho realised that the results of this paper might be used to construct a model of the quantum lambda calculus. I thank them, and Bart Jacobs, Sam Staton, Wim Veldman, and Bas Westerbaan for their help.

Funding was received from the European Research Council under grant agreement № 320571.

\section{References}

[1] Kenta Cho \& Abraham Westerbaan (2016): Von Neumann Algebras form a Model for the Quantum Lambda Calculus. arXiv:1603.02133v1 [cs.LO]

[2] Keith Devlin (1993): The joy of sets: fundamentals of contemporary set theory. Springer, doi:10.1007/978-14612-0903-4.

[3] Robert Furber \& Bart Jacobs (2013): From Kleisli categories to commutative $C^{*}$-algebras: Probabilistic Gelfand duality. In: Algebra and Coalgebra in Computer Science, Springer, pp. 141-157, doi 10.1007/978-3642-40206-7_12.

[4] Richard V. Kadison (1951): A representation theory for commutative topological algebra. 7, American Mathematical Society, doi:10.1090/memo/0007.

[5] Stephen Lack (2010): A 2-categories companion. In: Towards higher categories, Springer, pp. 105-191, doi:10.1007/978-1-4419-1524-5_4.

[6] Saunders Mac Lane (1998): Categories for the working mathematician. 5, springer, doi:10.1007/978-1-46129839-7

[7] B. Russo \& H. A. Dye (1966): A note on unitary operators in $C^{*}$-algebras. Duke Mathematical Journal 33(2), pp. 413-416, doi $10.1215 /$ S0012-7094-66-03346-1.

[8] W. Forrest Stinespring (1955): Positive functions on $C^{*}$-algebras. Proceedings of the American Mathematical Society 6(2), pp. 211-216, doi: $10.2307 / 2032342$.

[9] Stephen Willard (2004): General topology. Courier Dover Publications.

\section{A Additional Proofs}

Proof of Lemma 7 Define $L C:=F C$ for all objects $C$ of $\mathscr{K} \ell(U F)$ and

$$
L f:=\varepsilon_{F C_{2}} \circ F f
$$

for $f: C_{1} \longrightarrow U F C_{2}$ from $\mathbf{C}$. We claim this gives a functor $L: \mathscr{K} \ell(U F) \longrightarrow \mathbf{D}$.

(L preserves the identity) Let $C$ be an object of $\mathscr{K} \ell(U F)$, that is, an object of $\mathbf{C}$. Then the identity on $C$ in $\mathscr{K} \ell(U F)$ is $\eta_{C}$. We have $L\left(\eta_{C}\right)=\varepsilon_{F C} \circ F \eta_{C}=\operatorname{id}_{F C}$. 
(L preserves composition) Let $f: C_{1} \longrightarrow U F C_{2}$ and $g: C_{2} \longrightarrow U F C_{3}$ from $\mathbf{C}$ be given. We must prove that $L(g \odot f)=L g \circ L f$. We have:

$$
\begin{aligned}
L(g \odot f) & =L\left(\mu_{C_{3}} \circ U F g \circ f\right) & & \text { by def. of } g \odot f \\
& =\varepsilon_{F C_{3}} \circ F \mu_{C_{3}} \circ F U F g \circ F f & & \text { by def. of } L \\
& =\varepsilon_{F C_{3}} \circ F U \varepsilon_{F C_{3}} \circ F U F g \circ F f & & \text { by def. of } \mu_{C_{3}} \\
& =\varepsilon_{F C_{3}} \circ F g \circ \varepsilon_{F C_{2}} \circ F f & & \text { by nat. of } \eta \\
& =L g \circ L f & & \text { by def. of } L
\end{aligned}
$$

Hence $L$ is a functor from $\mathscr{K} \ell(U F)$ to $\mathbf{D}$.

Let us prove that $U \circ L=G$. For $f: C_{1} \longrightarrow U F C_{2}$ from $\mathbf{C}$ we have

$$
\begin{aligned}
U L f & =U\left(\varepsilon_{F C_{2}} \circ F f\right) & & \text { by def. of } L \\
& =U \varepsilon_{F C_{2}} \circ U F f & & \\
& =\mu_{C_{2}} \circ U F f & & \text { by def. of } \mu_{C_{2}} \\
& =G f & & \text { by def. of } G f .
\end{aligned}
$$

Let us prove that $L \circ V=F$. For $f: C_{1} \longrightarrow C_{2}$ from $\mathbf{C}$ be given, we have

$$
\begin{aligned}
L V f & =L\left(\eta_{C_{2}} \circ f\right) & & \text { by def. of } V \\
& =\varepsilon_{F C_{2}} \circ F \eta_{C_{2}} \circ F f & & \text { by def. of } L \\
& =F f & & \text { by counit-unit eq. }
\end{aligned}
$$

We have proven that there is a functor $L: \mathscr{K} \ell(U F) \rightarrow \mathbf{D}$ such that $U \circ L=G$ and $L \circ V=F$. We must still prove that it is as such unique.

Let $L^{\prime}: \mathscr{K} \ell(U F) \rightarrow \mathbf{D}$ be a functor such that $U \circ L^{\prime}=G$ and $L^{\prime} \circ V=F$. We must show that $L=L^{\prime}$. Let us first prove that $L^{\prime}$ and $L$ agree on objects. Let $C$ be an object of $\mathscr{K} \ell(U F)$, i.e., $C$ is an object of $\mathbf{C}$. Since $L^{\prime} \circ V=F$ and $V C=C$ we have $L^{\prime} C=L^{\prime} V C=F C=L C$. Now, let $f: C_{1} \rightarrow U F C_{2}$ from $\mathbf{C}$ be given (so $f$ is a morphism in $\mathscr{K} \ell(U F)$ from $C_{1}$ to $C_{2}$ ). We must show that $L^{\prime} f=L U \equiv \varepsilon_{F C_{2}} \circ F f$. Note that since $F$ is the left adjoint of $U$ there is a unique morphism $\bar{f}: F C_{1} \longrightarrow F C_{2}$ in $\mathbf{D}$ such that $U \bar{f} \circ \eta_{C_{1}}=f$. To prove that $L^{\prime} f=L f$, we show that both $L f$ and $L^{\prime} f$ have this property. We have

$$
\begin{aligned}
U L^{\prime} f \circ \eta_{C_{1}} & =G f \circ \eta_{C_{1}} & & \text { as } U \circ L^{\prime}=G \text { by assump. } \\
& =\mu_{C_{2}} \circ U F f \circ \eta_{C_{1}} & & \text { by def. of } G \\
& =\mu_{C_{2}} \circ \eta_{U F C_{2}} \circ f & & \text { by nat. of } \eta \\
& =f & & \text { as } U F \text { is a monad. }
\end{aligned}
$$

By a similar argument we get $U L f \circ \eta_{C_{1}}=f$. Hence $L f=L^{\prime} f$.

Proof of Theorem 9 We use the symbols from Notation 6 .

(i) $\Longrightarrow$ (ii) Suppose that $L$ is an isomorphism. We must prove that $F$ is bijective on objects. Note that $F=L \circ V$, so it suffices to show that both $L$ and $V$ are bijective on objects. Clearly, $L$ is bijective on objects as $L$ is an isomorphism, and $V: \mathbf{C} \longrightarrow \mathscr{K} \ell(U F)$ is bijective on objects since the objects of $\mathscr{K} \ell(U F)$ are those of $\mathbf{C}$ and $V C=C$ for all $C$ from $\mathbf{C}$. 
(ii) $\Longrightarrow$ (i) Suppose that (ii) holds. We prove that $L$ is an isomorphism by giving its inverse. Let $D$ be an object from $\mathbf{D}$. Note that since $F$ is bijective on objects there is a unique object $C$ from $\mathbf{C}$ such that $F D=C$. Define $K C:=D$.

Let $g: D_{1} \rightarrow D_{2}$ from $\mathbf{D}$ be given. Note that by definition of $K$ we have:

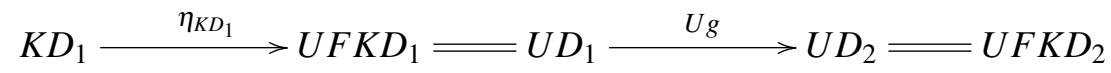

Now, define $K g: K D_{1} \rightarrow U F K D_{2}$ in $\mathbf{D}$ by $K g:=U g \circ \eta_{K D_{1}}$.

We claim that this gives a functor $K: \mathbf{D} \longrightarrow \mathscr{K} \ell(U F)$.

( $K$ preserves the identity) For an object $D$ of $\mathbf{D}$ we have

$$
\operatorname{Kid}_{D}=U \operatorname{id}_{D} \circ \eta_{K D}=\eta_{K D}
$$

and $\eta_{K D}$ is the identity on $K D$ in $\mathscr{K} \ell(U F)$.

(K preserves composition) Let $f: D_{1} \longrightarrow D_{2}$ and $g: D_{2} \longrightarrow D_{3}$ from $\mathbf{D}$ be given. We must prove that $K(g \circ f)=K(g) \odot K(f)$. We have

$$
\begin{aligned}
K(g) \odot K(f) & =\mu_{K D_{3}} \circ U F K g \circ K f & & \text { by def. of } \odot \\
& =\mu_{K D_{3}} \circ U F U g \circ U F \eta_{K D_{2}} \circ U f \circ \eta_{K D_{1}} & & \text { by def. of } K \\
& =U \varepsilon_{D_{3}} \circ U F U g \circ U F \eta_{K D_{2}} \circ U f \circ \eta_{K D_{1}} & & \text { by def. of } \mu \\
& =U g \circ U \varepsilon_{D_{2}} \circ U F \eta_{K D_{2}} \circ U f \circ \eta_{K D_{1}} & & \text { by nat. of } \varepsilon \\
& =U g \circ U f \circ \eta_{K D_{1}} & & \text { by counit-unit eq. } \\
& =K(g \circ f) & & \text { by def of } K .
\end{aligned}
$$

Hence $K$ is a functor from $\mathbf{D}$ to $\mathscr{K} \ell(U F)$. We will show that $K$ is the inverse of $L$. For this we must prove that $K \circ L=\mathrm{id}_{\mathbf{D}}$ and $L \circ K=\mathrm{id}_{\mathscr{K} \ell(U F)}$.

For a morphism $g: D_{1} \longrightarrow D_{2}$ from $\mathbf{D}$, we have

$$
\begin{aligned}
L K g & =L\left(U g \circ \eta_{K D_{1}}\right) & & \text { by def. of } K \\
& =\varepsilon_{F K D_{2}} \circ F U g \circ F \eta_{K D_{1}} & & \text { by def. of } L \\
& =g \circ \varepsilon_{F K D_{1}} \circ F \eta_{K D_{1}} & & \text { by nat. of } \varepsilon \\
& =g & & \text { by counit-unit eq. }
\end{aligned}
$$

For a morphism $f: C_{1} \longrightarrow U F C_{2}$ in $\mathbf{C}$ we have

$$
\begin{aligned}
K L f & =K\left(\varepsilon_{F C_{2}} \circ F f\right) & & \text { by def. of } L \\
K L f d d & =U \varepsilon_{F C_{2}} \circ U F f \circ \eta_{K F C_{1}} & & \text { by def. of } K \\
& =U \varepsilon_{F C_{2}} \circ \eta_{U F C_{2}} \circ f & & \text { by nat. of } \eta \\
& =f & & \text { by counit-unit eq. }
\end{aligned}
$$

Hence $K$ is the inverse of $L$, so $L$ is an isomorphism. 